\title{
A Real-Time Method to Measure the Deviation of Deep Hole
}

\author{
Junqiang Liu, Fujia Wu, Xiaobing Gao and Ruicheng Li \\ School of Mechatronic Engineering, Xi'an Technological University, Xi' an 710032, China
}

\begin{abstract}
The deviation of deep hole's axis is measured in real-time machining process by using ultrasonic thickness measurement method. A ultrasonic device is designed using 3-points circle method, the polar parameter model of measurement objects is built and the function relationship between object and measured data is established. Grubbs criteria is used to remove the gross error of data aim to the unstable of online measurement. Using error limits is raised to estimate measuring error. It is verified by actual measurement that this method can measure the deviation and its direction of deep hole's axis accurately in real-time machining process and the measurement error can be controlled under the 2 times of single probe error.
\end{abstract}

Keywords-deep hole machining; ultrasonic thickness measurement; deviation of deep hole; error limits

\section{INTRODUCTION}

The definition of hole's axis deviation is its axis straightness error, correspond to the deviation between actual axis of machined hole and theoretical axis. The deviation of hole's axis is the most important index of its precision, and is also the most instable quality element in many processing quality elements of deep hole[1]. According to GB/T1182-1996, straightness error is the variation between actual axis and ideal axis and is used to control the shape error of planar or spatial line. The shape of straightness's tolerance variation is composed of given plane, given direction and random direction. The deviation of hole's axis is belong to random direction error. It is difficult to build a precise mathematical model of deep hole's machining process because it is so complex that there are many factors such as high clearance of guide sleeve, low stiffness of drill pipe, non-uniform of workpiece's material, the dissymmetry of cutter's shape and the machine tool's coaxiality in assembly[2]. In practice, most deviation of deep hole's axis will appear on the moment when drill come inside workpiece and it will increase with the deep of hole. Because deep hole's drilling process often takes a long time, so the deviation and the reject rate of machining can be reduced if a real-time measurement method be taken to monitor the process of drilling and the adjustment will be taken when the tendency of deviation appears $[3,4]$.

Due to most deep holes has the characteristics as great length, small aperture, So the space that could be used to detect straightness of deep hole directly is very limited, and the operation of straightness inspection is difficult. At present, there are 9 commonly used deep hole's straightness inspection methods: straightness gauge method, leverage measurement method, gas measurement instruments method, alignment telescope measurement method, laser alignment method, ultrasonic thickness measurement method. Although straightness gauge method and leverage measurement method are easy to operate, they have common disadvantages: low automation level, low measurement precision and limitation of measurement place, so they always use to measure the straightness of shallow holes with low precision[5].The features of gas measurement instruments method are: high measuring precision, can be used for non-contact measurement, less measuring pressure, favorable to thin-wall part's measurement, suitable for mass production measurement, measuring tool has simple structure, low requirements for environment, can be used automatic measurement[6]. Alignment telescope measurement method has a simple structure, it's advantage is: convenient operation, low cost, wide application range, high precision, but it is only suitable for measuring processing good hole. Laser method alignment is convenient to aim, high efficiency, high measurement precision and easy to realize automation[7]. All above methods are suitable to measure the machined deep hole, that is the measurement only can be used after deep holes formation, so it often unable to measure holes during the forming process of them. Ultrasonic thickness measurement method is advanced and practical due to its instrument is small in size, measurement results stable, fast measurement and high accuracy. Its measurement precision mainly related to the resolution of the thickness gauge display value. It can realize online measurement in the process of machining and can satisfy the requirement of high precision measurement.

\section{Ultrasonic ThickNess MEASUREMENT MEthoD}

\section{A. The Theory of Ultrasonic Thickness Measurement}

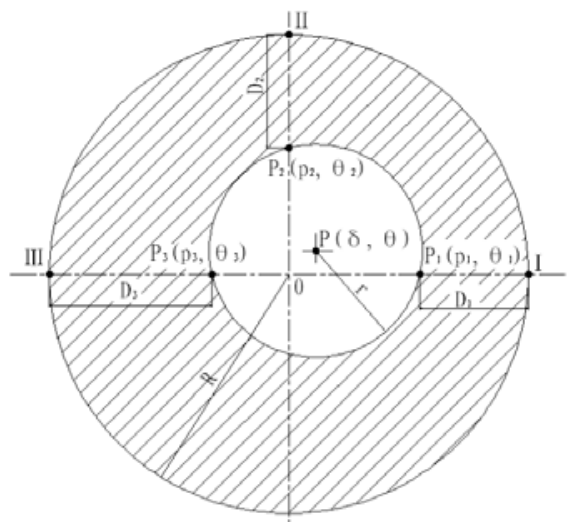

FIGURE I. SCHEMATIC DIAGRAM UNDER POLAR COORDINATE OF MEASURING DEVIATION 
The velocity of ultrasonic wave in the same medium is constant, it has reflection characteristics when the wave travels to the different medium interface. This characteristics can be used to measure the thickness of machined hole. According to those thickness which distribute in the fixed position of workpiece, the axis straightness error of hole can be calculated. Suppose the velocity of ultrasonic wave in certain medium is C, the time difference that reflection wave travels through workpiece inside and outside surface is $t$, the thickness of workpiece d can calculated as:

\section{$\mathrm{d}=\mathrm{ct} / 2$}

According to the principle of three-point determine a circle, it required to measure at least 3 points on the circumference of workpiece.

\section{B. The Arrangement of Probes}

There are 3 ultrasonic probes around circle to detect the thickness of the 3 points in detection device. Considering the convenience of online measurement, the device is installed in horizontal deep hole drilling \& boring machine which is machining cylindrical surfaces. Machine tool coordinate system is regarded as measurement coordinate system, The coordinates is defined as follow:

$\mathrm{Z}$ axis: the spindle direction,

$\mathrm{X}$ axis: horizontal direction

Y axis: vertical direction.

By measuring hole's wall thickness in different positions on the $\mathrm{Z}$ axis, the hole axis deflection in the location can be calculated. If the amount of the deviation exceeded the set threshold value, in order to prevent the excessive deflection in machining error, a correct operation will be used to force cutter to stretch the opposite direction. All measure plane are consists of $\mathrm{X}, \mathrm{Y}$ axis, Testing point I and III are arranged on the positive and negative direction of $\mathrm{X}$ axis, and a testing point II in the Y. The incident direction of ultrasonic wave is the normal direction of workpiece's cylindrical surface. The schematic diagram under polar coordinate of measuring deviation is shown in figure 1 . The meaning of the symbols in as follow:

$\mathrm{R}$ : the cylindrical radius of workpiece

$\mathrm{r}:$ the drilled deep hole's radius

$D_{\mathrm{i}}$ : wall thickness at testing point $\mathrm{i}$

$\delta$ : the deviation of hole

$\theta$ : the direction of deviation

$P(\delta, \theta)$ : the polar coordinate of hole's center

$P_{i}\left(p_{i}, \theta_{i}\right)$ : the polar coordinate of test point $P_{i}$

here: $\theta_{1=0^{\circ},} \theta_{2=90^{\circ}}, \theta_{3=180^{\circ}}$

\section{Method of Calculation}

$P_{1}\left(p_{1}, \theta_{1}\right), P_{2}\left(p_{2}, \theta_{2}\right), P_{3}\left(p_{3}, \theta_{3}\right)$ distribute on the circumference of a circle whose radius is $\mathrm{r}$, so:

$$
\left|P_{1} P\right|=\left|P_{2} P\right|=\left|P_{3} P\right|=r
$$

$$
\begin{aligned}
& p_{1}^{2}+\delta^{2}-2 p_{1} \delta \cos \left(\theta_{1}-\theta\right)=p_{3}^{2}+\delta^{2}-2 p_{2} \delta \cos \left(\theta_{3}-\theta\right) \\
& p_{1}^{2}+\delta^{2}-2 p_{1} \delta \cos \left(\theta_{1}-\theta\right)=p_{2}^{2}+\delta^{2}-2 p_{2} \delta \cos \left(\theta_{2}-\theta\right)
\end{aligned}
$$

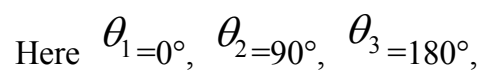

$$
\begin{aligned}
& p_{1}=R-D_{1} \\
& p_{2}=R-D_{2} \\
& p_{3}=R-D_{3}
\end{aligned}
$$

Equation (2) can be simplified to:

$$
\begin{gathered}
\theta=\arctan \frac{p_{2}^{2}-p_{1} p_{3}}{p_{2}\left(p_{1}-p_{2}\right)} \\
\delta=\frac{p_{1}-p_{3}}{2 \cos \theta}
\end{gathered}
$$

\section{Treatment of Measured Data}

\section{A. Elimination of Gross Error}

Grubbs rule is used to eliminate gross error of measured data.

According to Grubbs rule, there is a group of data $\mathrm{Xi}$ which is continuous and has the same accuracy, if $\left|X_{d}-\bar{X}\right|>\lambda(\alpha, n) S$, then eliminate $X_{d}$ from the group.

Here $\bar{X}=\frac{1}{n} \sum_{i=1}^{n} X_{i}$ is the mean value of the serials of data is $S=\sqrt{\frac{\sum_{i=1}^{n}\left(X_{i}-\bar{X}\right)^{2}}{n-1}}$ the standard deviation of the serials of data.

$\lambda$ is the critical value of Grubbs rule, it is decided by $n$-the times

$\alpha$-Significance level of test

\section{B. Estimation of System Error in Measurement}

The relation of object value and tested value is complex in Eq. 3 and Eq.4, so it is difficult to infer the transfer of errors by using traditional error calculation formula. A geometric error limitation method is used here to estimate the error limitation of the hole's axis deviation value by building the limited error of thickness measured directly in 3 points. There are 3 same 
ultrasonic thickness measurement devices around the tested piece, According to the principle of error is similar, the measurement error of those 3 devices is same, that is the measurement error of them all are $\pm \triangle$. The 2 limited error positions can be set in every measurement point, so there are 6 limited error positions in all. Select any position from each point as circle's constructional point, $23=8$ circles can be built from those 6 limited error positions, those circles can be called the limited circles. The limited system error is decided by the offset between the center of limited circle and original point in measured length direction. It can be calculated by geometric method that the maximal error is $\frac{2 r}{r-\delta} \Delta$ and system error in measurement can be estimated by them.

\section{Measurement ExAmple}

A tube piece machined in BTA inner chip removal deep hole machine tools was measured by the device, parameters are as follows: the outside diameter of piece: $50.12 \mathrm{~mm}$, length of piece: $1600 \mathrm{~mm}$, the diameter of deep hole: $\Phi 18.4 \mathrm{~mm}$, range of thickness measurement of ultrasonic device: $0.15 \sim 25.4 \mathrm{~mm}$. Adjustable sound velocity range: $1250 \sim 10000 \mathrm{~m} / \mathrm{s}$, measurement accuracy: $\pm 0.005 \mathrm{~mm}$.

There are 10 thickness data tested at 3 points whose length is $250 \mathrm{~mm}$ is showed as table 1 . When significance level of test $\alpha$ is $0.05, \lambda(0.05,10)=2.176$. The measured data are determined by Grubbs rule, D1 and D2 are qualified, the first datum D3 is not qualified and is deleted in D3, the rest data in D3 is are determined and qualified. The processed the data as shown in table 2.

TABLE I. ORIGINAL DATA OF THICKNESS

\begin{tabular}{|c|c|c|c|}
\hline & $\mathrm{D}_{1}(\mathrm{~mm})$ & $\mathrm{D}_{2}(\mathrm{~mm})$ & $\mathrm{D}_{3}(\mathrm{~mm})$ \\
\hline 1 & 20.651 & 20.665 & 20.426 \\
\hline 2 & 20.642 & 20.668 & 20.439 \\
\hline 3 & 20.631 & 20.672 & 20.456 \\
\hline 4 & 20.643 & 20.661 & 20.457 \\
\hline 5 & 20.647 & 20.659 & 20.451 \\
\hline 6 & 20.649 & 20.667 & 20.448 \\
\hline 7 & 20.638 & 20.657 & 20.458 \\
\hline 8 & 20.639 & 20.675 & 20.453 \\
\hline 9 & 20.645 & 20.664 & 20.451 \\
\hline 10 & 20.641 & 20.652 & 20.459 \\
\hline Mean & 20.643 & 20.664 & 20.450 \\
\hline S & 0.006 & 0.007 & 0.010 \\
\hline
\end{tabular}

TABLE II. VALID DATA AFTER PROCESS

\begin{tabular}{|l|l|l|l|}
\hline & $\mathrm{D}_{1}(\mathrm{~mm})$ & $\mathrm{D}_{2}(\mathrm{~mm})$ & $\mathrm{D}_{3}(\mathrm{~mm})$ \\
\hline 1 & 20.651 & 20.665 & \\
\hline 2 & 20.642 & 20.668 & 20.439 \\
\hline 3 & 20.631 & 20.672 & 20.456 \\
\hline 4 & 20.643 & 20.661 & 20.457 \\
\hline 5 & 20.647 & 20.659 & 20.451 \\
\hline 6 & 20.649 & 20.667 & 20.448 \\
\hline 7 & 20.638 & 20.657 & 20.458 \\
\hline 8 & 20.639 & 20.675 & 20.453 \\
\hline 9 & 20.645 & 20.664 & 20.451 \\
\hline 10 & 20.641 & 20.652 & 20.459 \\
\hline Mean & 20.643 & 20.664 & 20.452 \\
\hline S & 0.006 & 0.007 & 0.006 \\
\hline
\end{tabular}

The 3 mean values of thickness are regarded as the final measure thickness, the radius of piece's outer circle $\mathrm{R}=25.06$, so it can be calculated:

$$
\mathrm{p} 1=4.417, \mathrm{p} 2=4.396, \mathrm{p} 3=4.61
$$

According to above results and Eq.3 and Eq.4, draw the following conclusions:

$$
\begin{aligned}
& \theta=-140.72^{\circ} \\
& \delta=0.152 \mathrm{~mm}
\end{aligned}
$$

Due to an indirect measurement method was adopted in the measurement device, measurement error can be transmitted. So an error limitation method was taken to estimate error. There are 3 kinds of error affect the measurement result: the measurement error of piece's outer circle $\Delta 1$, the roundness error of hole $\Delta 2$ and the measurement error of hole's deviation $\Delta 3$. They are independent each other. It can tested that $\Delta 1= \pm 0.001 \mathrm{~mm}, \Delta 2= \pm 0.001 \mathrm{~mm}$. Here $r=4.515 \mathrm{~mm}$, $\delta=0.152 \mathrm{~mm}, \Delta= \pm 0.005 \mathrm{~mm}$.

$$
\Delta_{3}= \pm \frac{2 \mathrm{r} \delta}{\mathrm{r}-\delta} \Delta= \pm 0.01 \mu \mu
$$
by

It can estimated the total error of the measurement system

$$
\Delta=\sqrt{\Delta_{1}{ }^{2}+\Delta_{2}{ }^{2}+\Delta_{3}{ }^{2}}= \pm 0.01 \mathrm{~mm}
$$

It is 2 times of single ultrasonic probe measurement precision.

\section{SUMMARY}

A ultrasonic thickness measurement method in 3-points of circumference is used in the paper to locate the center of circle, and make further to determine the deviation of deep hole's axis during the processing of machining. The polar parameter model of measurement objects is built and the function relationship between object and measured data is established. Grubbs criteria is used to remove the gross error of data aim to the unstable of online measurement., a new geometric method using error limits is raised to establish measuring error. It is verified by actual measurement that this method can measure the deviation and its direction of deep hole's axis accurately in real-time machining process and the measurement error can be controlled under the 2 times of single probe error.

\section{ACKNOWLEDGEMENTS}

The paper is sponsored by shaanxi province industrial science and technology research projects (2015GY061) .

\section{REFERENCES}

[1] Wang Jun. Modern deep hole machining technology. Harbin: Harbin industrial university press,2005:1-2 
[2] Robert Heinemann,Sri Hinduja.A new strategy for tool condition monitoring of small diameter twist drills in deep-hole drilling[J]. International Journal of Machine Tools \&Manufacture 52 (2012) 69-76.

[3] Akio Katsuki, Hiromichi Onikura, Takao Sajima. Development of a practical high performance laser-guided deep-hole boring tool: Improvement in guiding strategy[J].Precision Engineering, $2011,35(4): 221-227$.

[4] K. Weinert,O. Webber,A. Experimental Investigations of the Dynamics of the BTA Deep Hole Drilling Process[J],Journal of Production Engineering-Research Development Germany,2009, 45 (2):925-935.

[5] Wang Ning. The design and application of deep hole straightness inspection device [J]. Mechanical design and manufacturing,2007, (2): 90- 92.

[6] Zhu Daili, Ren chengao, Ren Dong. The measurement system of deep hole straightness based on the three point method [J]. Equipment manufacturing technology, 2009(11):79-81.

[7] Wang NingXia. The design and application of deep hole straightness inspection device $[\mathrm{J}]$. Mechanical design and manufacturing,2007(2):12-13. 